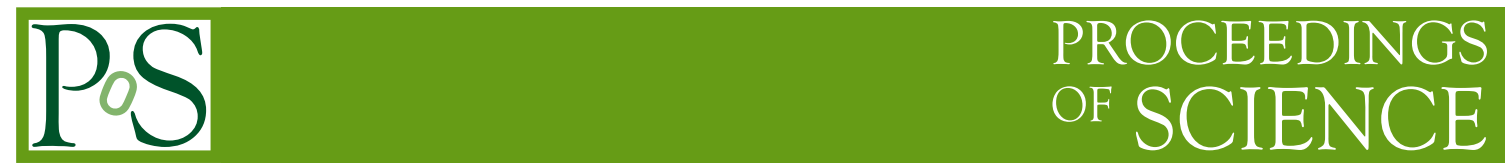

\title{
Young Black Holes Accreting From Fallback Disks
}

\section{K. Yavuz Ekşi ${ }^{*}$}

Istanbul Technical University, Faculty of Science and Letters,

Dept. Of Physics Engineering, Maslak 34469, Istanbul, TURKEY

E-mail: eksi@itu.edu.tr

\begin{abstract}
The prospects for the formation of a long lived fallback disk around a stellar mass black hole following a supernova explosion is discussed. The evolution of the disk is described in terms of its initial mass and angular momentum. The luminosity of the disk at the age of $\sim 10^{4}-10^{5}$ years is calculated. It is argued that a few such black holes can be detected in persistent states in the Galaxy, either in radiatively efficient or radiatively inefficient state. Fallback disks will have higher abundance of iron than disks in binary systems. This could make them suitable candidates for studying the strong gravity effects by measuring the Fe $\mathrm{K}$ lines.
\end{abstract}

VII Microquasar Workshop: Microquasars and Beyond September 1-5 2008

Foca, Izmir, Turkey

${ }^{*}$ KYE acknowledges support from TÜBİTAK. 


\section{INTRODUCTION}

Stellar mass black holes (BHs) form in the core collapse of massive stars. Some of the ejected matter may remain bound to the gravitational potential of the system and fall back [1,2]. As the progenitors of stellar mass BHs are rapidly rotating massive stars, the fallback matter may carry sufficient angular momentum to form a fallback disk [3, 4].

In the "collapsar" model [5, 6] of gamma ray bursts (GRBs) the central engine is a BH rapidly accreting from a torus formed by fallback matter. After this brief episode of rapid accretion in which accretion proceeds first via neutrino cooling [7] and then by photon cooling [8], the mass flow rate $\dot{M}$ drops below the Eddington limit and the thin disk regime [9] prevails. Finally, when $\dot{M}$ drops below $\sim 0.01 \dot{M}_{\text {Edd }}$, the disk becomes an optically thin advection dominated accretion flow (ADAF) [10].

During the early accretion processes mass of the disk declines very rapidly while the angular momentum of the disk remains nearly constant, increasing the average specific angular momentum of the disk. The mass leftover from the rapid accretion episode and the enhanced specific angular momentum determine the initial conditions for the thin disk regime.

Mineshige et al. [11] studied fallback disks around BHs in the slim-disk regime and suggested that the hyphothetical $\mathrm{BH}$ in $\mathrm{SN}$ 87A is accreting from such a disk. Li [12] suggested that the ultraluminous X-ray sources (ULXs) associated with supernova remnants are BHs accreting from fallback disks which are descendants of fallback disks of GRBs.

The detection of a debris disk around a young neutron star [13] rekindled interest in possible implications of fallback disks[14, 15]. The presence of fallback disks around such young neutron stars was suggested by [16] and [17]. In an attempt to address the elusivity of fallback disks around neutron stars [18] argued that the rapidly rotating magnetosphere of a neutron star could blow away the disk and suggested that fallback disks would more likely survive around BHs.

In the present study the evolution of a viscous thin disk is described by its initial mass and angular momentum. The luminosity of a fallback disk around a stellar mass black hole is calculated for a reasonable range of initial mass and angular momentum. It is shown that even at the age of $10^{4}$ years the disk can be in the thin disk regime.

\section{MASS AND ANGULAR MOMENTUM BUDGET OF THE DISK}

The progenitors of BHs are massive stars rotating very rapidly with a surface speed of $\sim$ $250-400 \mathrm{~km} \mathrm{~s}^{-1}$ [19]. The specific angular momentum of matter on the surface is $j \sim 10^{19} \mathrm{~g} \mathrm{~cm}^{2}$. The origin of the fallback matter is the inner part of the layers outside the collapsing core. The specific angular momentum of this region should be less than $j=10^{19} \mathrm{gcm}^{2}$.

The angular momentum distribution inside the star is a subject of debate but we can estimate a minimum value for the specific angular momentum of the fallback matter. A maximally rotating BH will have an angular momentum of $J_{\max }=G M_{\bullet}^{2} / c=8.8 \times 10^{50}\left(M_{\bullet} / 10 M_{\odot}\right)^{2}$ corresponding to a specific angular momentum of $j=4.4 \times 10^{16}\left(M_{\bullet} / 10 M_{\odot}\right) \mathrm{gcm}^{2}$. It is expected that a newborn BH is close to this maximal rotation meaning that $j \sim 10^{16} \mathrm{~g} \mathrm{~cm}^{2}$ is a reasonable average specific angular momentum for the core. Outermost part of the collapsing core should have a larger specific angular momentum than this average value, possibly $j \sim 10^{17}-10^{18} \mathrm{gcm}^{2}$. The inner part of the 
layers outside the collapsing core, the origin of the fallback matter, should have specific angular momentum in this range possibly favoring the higher end.

Woosley \& Heger [20] calculated the specific angular momentum distribution inside massive stars just before the supernova explosion. Figure 2 of their paper shows that $j \sim 10^{18} \mathrm{~g} \mathrm{~cm}^{2}$ right outside the sphere containing $11 M_{\odot}$.

The mass of the fallback matter is not very well determined by the simulations but can be in the wide range of $0.1-5 M_{\odot}$ [5]. After the initial rapid mass accretion stage, the mass of the matter left can be as small as $10^{-5}-10^{-2} M_{\odot}$. The material with the lowest angular momentum is accreted in the first few seconds while the total angular momentum of the disk remains almost constant. When $\dot{M}$ drops to the Eddington limit, $j$ can be as high as $10^{21} \mathrm{~g} \mathrm{~cm}^{2}$.

\section{FORMATION AND EVOLUTION OF THE DISK}

The disk can form if the circularization radius $R_{\text {circ }}=j^{2} / G M \bullet$ of the leftover fallback material is greater than the innermost circular stable orbit (ISCO) which is equal to the Schwarzschild radius $R_{s}=2 G M_{\bullet} / c^{2}$ for a maximally rotating $\mathrm{BH}$ or $3 R_{s}$ for a non-rotating $\mathrm{BH}$. The circularization radius in terms of $R_{S}$ is

$$
\frac{R_{\text {circ }}}{R_{\mathrm{S}}} \simeq 250\left(\frac{j}{10^{18}}\right)^{2}\left(\frac{M_{\bullet}}{10 M_{\odot}}\right)^{-2}
$$

which shows that formation of a disk is highly feasible as long as $j \gtrsim 10^{17} \mathrm{gcm}^{2}$.

The viscous evolution of a thin disk with keplerian angular velocity, $\Omega_{\mathrm{K}}=\sqrt{G M / r^{3}}$, is described by the diffusion equation

$$
\frac{\partial \Sigma}{\partial t}=\frac{3}{r} \frac{\partial}{\partial r}\left[r^{1 / 2} \frac{\partial}{\partial r}\left(v \Sigma r^{1 / 2}\right)\right]
$$

where $\Sigma$ is the surface mass density and $v$ is the turbulent viscosity. For a general dependence of the form $v=C r^{p} \Sigma^{q}$, where $C, p$ and $q$ are constants, the equation is nonlinear.

Following [21] we calculate the evolution of the disk by employing the Pringle solutions [22]. As Equation(3.2) is symmetric under $t \rightarrow t+t_{0}$ we modified the original solutions by a time shift so that the solution does not diverge at $t=0$. Of course this does not solve the initial value problem but pushes the problematic point out of the domain of interest. We have compared the time-shifted analytical solution with a numerical solution and found that the shifted solution is remarkably succesful (see [23]). Of course neither the original solitions nor the time-shifted solutions can accomodate the inner boundary condition, a characteristic problem of a self-similar solution. Thus the solution we use is

$$
\frac{\Sigma}{\Sigma_{0}}=k\left(1+\frac{t}{t_{0}}\right)^{-\frac{5}{5 q-2 p+4}}\left(\frac{r}{R_{\text {out }}(t)}\right)^{-\frac{p}{q+1}}\left[1-\left(\frac{r}{R_{\text {out }}(t)}\right)^{2-\frac{p}{q+1}}\right]^{1 / q}
$$

where $R_{\text {out }}(t)=r_{0}\left(1+t / t_{0}\right)^{2 /(5 q-2 p+4)}$ and $k=\left(\frac{q}{(4 q-2 p+4)(5 q-2 p+4)}\right)^{1 / q}$. The mass flow rate of the disk declines as

$$
\dot{M}_{\mathrm{d}}=\dot{M}_{0}\left(1+\frac{t}{t_{0}}\right)^{-\alpha}
$$



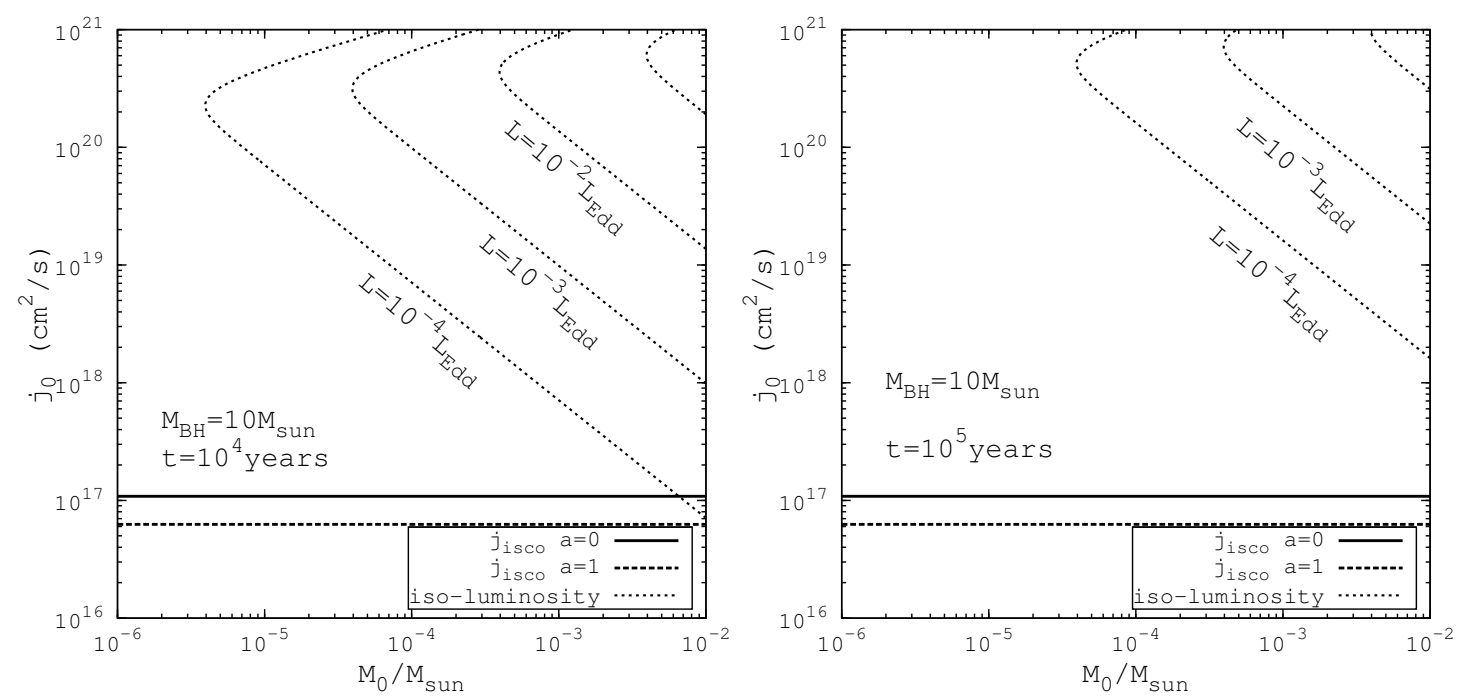

Figure 1: The iso-luminosity lines for a range of initial mass and angular momentum around a $\mathrm{BH}$ with mass $10 M_{\odot}$ at an age of $t=10^{4}$ years (left panel) and at an age of $t=10^{5}$ years (right panel). The horizontal lines are the specific angular momentum corresponding to the innermost stable orbit, $j_{\text {isco }}=\sqrt{G M R_{\mathrm{isco}}}$, of a non-rotating $(\mathrm{a}=0)$ and maximally rotating $(\mathrm{a}=1) \mathrm{BH}$.

where $\alpha=19 / 16$ for a disk dominated by electron scattering opacity and $\dot{M}_{0}=(\alpha-1) M_{0} / t_{0}$ (see [21] for details).

For a disk of initial mass $M_{0}$ and angular momentum $J_{0}$ the other scale factors can be found through

$$
\begin{aligned}
r_{0} & =\left(\frac{\gamma_{2}}{\gamma_{1}}\right)^{2} \frac{\left(J_{0} / M_{0}\right)^{2}}{G M_{\bullet}} \\
\Sigma_{0} & =\frac{M_{0}}{4 \pi r_{0}^{2} \gamma_{2}} \\
v_{0} & =C r_{0}^{p} \Sigma_{0}^{q} \\
t_{0} & =4 r_{0}^{2} / 3 v_{0}
\end{aligned}
$$

where $\gamma_{1}=3.685 \times 10^{-4}$ and $\gamma_{2}=6.263 \times 10^{-4}$ for electron scattering opacity (see [23] for detail). Thus, the evolution of the disk is determined given the initial mass and angular momentum of the disk.

\section{RESULTS}

We have calculated the luminosity of the disk $L=\eta \dot{M}_{\mathrm{d}} c^{2}$ where the efficiency factor $\eta \sim 0.1$ depends on the spin of the BH. Figure 1 shows the iso-luminosity lines for a range of initial mass, $M_{0}$ and specific angular momentum $j_{0}$ of the disk.

It is seen that at $10^{4}$ years the disk can still be in the thin disk regime if the leftover mass is greater than $10^{3} M_{\odot}$ after the initial brief rapid mass accretion stage. The disk is most likely in an ADAF phase [10] at the age of $10^{5}$ years for most reasonable mass and angular momentum ranges. 


\section{DISCUSSION}

We presented the evolution of a viscous thin thin disk in terms of initial mass and angular momentum. We argued that the initial rapid mass accretion stage increases the average specific angular momentum of the disk. We have seen that the disk can still be in the radiatively efficient thin disk regime if the initial mass of the thin disk after initial rapid accretion stage is greater than $10^{4}$ years.

How many $\mathrm{BH}$ can we expect to be accreting from a fallback disk in our galaxy? If the supernova rate per galaxy is 100 years then there can be about 100 explosions with ages less than $10^{4}$ years. If only 10 per cent of these supernova explosions produce $\mathrm{BHs}$ then there could be 10 $\mathrm{BH}$ accreting from a fallback disk in our galaxy.

As a final note we would like to stress that fallback disks will have high abundance of iron and heavy elements compared to disks in binary systems. This makes them suitable candidates for studying the strong gravity effects by measuring the Fe $\mathrm{K}$ lines.

\section{References}

[1] S. A. Colgate, Neutron-Star Formation, Thermonuclear Supernovae, and Heavy-Element Reimplosion, ApJ, 163, 221 (1971).

[2] R. A. Chevalier, Neutron star accretion in a supernova, ApJ 346, 847 (1989).

[3] F. C. Michel \& Dessler, A. J., Pulsar disk systems, ApJ 251, 654 (1981).

[4] D. N. C. Lin, Woosley, S. E., \& Bodenheimer, P. H., Formation of a planet orbiting pulsar 1829 - 10 from the debris of a supernova explosion, Nature 353, 827 (1991).

[5] S. E. Woosley, Gamma-ray bursts from stellar mass accretion disks around black holes, ApJ, 405, 273 (1993).

[6] A. I. MacFadyen, \& Woosley, S. E., Collapsars: Gamma-Ray Bursts and Explosions in "Failed Supernovae”, ApJ 524, 262 (1999).

[7] R. Narayan, Piran, T., \& Kumar, P., Accretion Models of Gamma-Ray Bursts, ApJ 557, 949.

[8] M. A. Abramowicz, Czerny, B., Lasota, J. P., \& Szuszkiewicz, E., Slim accretion disks, ApJ 332, 646.

[9] N. I. Shakura \& Sunyaev R., Black holes in binary systems: Observational appearance, A\&A 24, 337.

[10] R. Narayan, \& Yi, I., Advection-dominated accretion: A self-similar solution, ApJ 428, L13.

[11] S. Mineshige, Nomura, H., Hirose, M., Nomoto, K., \& Suzuki, T., Black Hole Disk Accretion in Supernovae, ApJ, 489, 227 (1997).

[12] X.-D. Li, A Fallback Disk Model for Ultraluminous X-Ray Sources, ApJ 596, L199 (2003).

[13] Z. Wang, Chakrabarty, D. \& Kaplan, D. L., A debris disk around an isolated young neutron star, Nature 440, 772 (2006).

[14] Ü. Ertan, Erkut, M. H., Ekşi, K. Y. \& Alpar, M. A., The Anomalous X-Ray Pulsar 4U 0142+61: A Neutron Star with a Gaseous Fallback Disk, ApJ 657, 441 (2007).

[15] P. B. Jones, Interaction and ablation of fall-back discs in isolated neutron stars, MNRAS 382, 871 (2007). 
[16] P. Chatterjee, Hernquist, L., \& Narayan, R., An Accretion Model for Anomalous X-Ray Pulsars, ApJ 534, 373 (2000).

[17] M. A. Alpar, On Young Neutron Stars as Propellers and Accretors with Conventional Magnetic Fields, ApJ 554, 1245 (2001).

[18] K. Y. Ekşi, , Hernquist, L., \& Narayan, R., Where Are All the Fallback Disks? Constraints on Propeller Systems, ApJ 623, L41 (2005).

[19] R. H. D. Townsend, , Owocki, S. P., \& Howarth, I. D., Be-star rotation: how close to critical?, MNRAS 350, 189 (2004).

[20] S. E. Woosley \& Heger, A., The Progenitor Stars of Gamma-Ray Bursts, ApJ 637, 914 (2006).

[21] J. K. Cannizzo, Lee, H.M. \& Goodman, J., The disk accretion of a tidally disrupted star onto a massive black hole, ApJ 351, 38 (1990).

[22] J. E. Pringle, Accretion and Binary X-ray Sources, PhD thesis, Univ. Cambridge (1974).

[23] Ü. Ertan, Ekşi, K. Y., Erkut, M. H. \& Alpar, M. A., On the Evolution of Anomalous X-ray Pulsars and Soft Gamma Ray Repeaters with Fallback Disks, ApJ in preperation. 